\title{
\begin{tabular}{l|l} 
POLITIQUES \& & Politiques et management public
\end{tabular}

\section{La dérégulation endogène du système français de médecine libérale}

The Internal Deregulation of Physicians in Private Practice in the French Medical System

\section{Francis Pavé}

\section{CpenEdition}

\section{Journals}

Electronic version

URL: http://journals.openedition.org/pmp/4876

ISSN: $2119-4831$

Publisher

Institut de Management Public (IDPM)

\section{Printed version}

Date of publication: 15 June 2012

Number of pages: 191-213

ISBN: 978-2-7430-1470-4

ISSN: 0758-1726

\section{Electronic reference}

Francis Pavé, "La dérégulation endogène du système français de médecine libérale », Politiques et management public [En ligne], Vol 29/2 | 2012, mis en ligne le 10 octobre 2014, consulté le 01 mai 2019. URL : http://journals.openedition.org/pmp/4876 


\title{
La dérégulation endogène du système français de médecine libérale
}

\author{
Francis Pavé \\ Chercheur CNRS, Laboratoire CSO \\ CSO Sciences Po - 19, rue Amélie - 75007 Paris
}

\section{Résumé}

L'observation du fonctionnement des médecins libéraux en France, tant généralistes que spécialistes, révèle qu'ils cherchent à neutraliser les régulations qu'on veut leur imposer. Ce qui ouvre autant de questions quant aux modes d'action mis en œuvre. Cette dérégulation est endogène, non concertée et liée à l'individualisme foncier des médecins, dont les comportements néanmoins convergent et permettent de constituer une stratégie collective efficace, tant au niveau de leurs pratiques professionnelles qu'institutionnelles.

(c) 2012 IDMP/Lavoisier SAS. Tous droits réservés

Mots clés : santé, médecine libérale, sécurité sociale, syndicalisme, lobby, régulation.

\section{Abstract}

The Internal Deregulation of Physicians in Private Practice in the French Medical System. Observation of doctors in private practice in France, general practitioners as well as specialists, shows that they avoid regulation. How do they do it? This is an internal affair of deregulation, one that has taken place without discussion and which is a symptom of the fundamental individualism of doctors. However, across the board, doctors tend to behave and act in similar ways, which would point to the possibility of creating effective collective strategies, both in terms of their professional and institutional practices.

(c) 2012 IDMP/Lavoisier SAS. Tous droits réservés

Keywords: Health, Physicians in Private Practice, French Public Welfare System, Syndicalism, Lobby, Regulation.

*Auteur correspondant : francis.pave@sciences-po.fr

doi:10.3166/pmp.29.191-213 @ 2012 IDMP/Lavoisier SAS. Tous droits réservés 


\section{Introduction}

Les médecins sont classifiés par la sociologie anglo-saxonne et tout particulièrement par (Eliot Freidson, 1984) comme des « Professionnels », ce qui leur confère une « habilitation » à se gouverner eux-mêmes en tant que groupe socio-économique. En France, l'indépendance professionnelle des médecins est reconnue comme un principe général du droit (Tabuteau, 2010 : 1). Par ailleurs, la profession est largement impliquée dans la réglementation de son propre exercice, à telle enseigne qu'on a pu la qualifier de «profession législatrice » (Tabuteau, $2010: 9-11$ ). Elle dispose d'un Ordre qui participe pleinement à cette tâche et qui autorise l'exercice des praticiens, une fois vérifié que les médecins ont bien suivi un cursus de formation complet, soutenu leur doctorat en médecine et qu'ils connaissent les règles de la profession. Tout médecin doit être inscrit à l'Ordre de son département d'exercice et tout patient peut saisir l'Ordre s'il s'estime mal traité.

L'Ordre est en charge de la déontologie médicale, de son évolution et de son respect : il existe une chambre disciplinaire qui juge les médecins ayant fait l'objet d'une plainte et qui peut, le cas échéant, interdire à un médecin d'exercer. L'ordre est donc l'instance première de régulation de ces professionnels. Or nous verrons que c'est loin d'être le cas. Toutefois, avec la mise en place de la Sécurité Sociale à la fin de la guerre, le dispositif de régulation s'est compliqué et met en défaut l'autonomie revendiquée par la profession.

La Caisse Nationale d'Assurance-maladie des travailleurs salariés (CNAMts) ${ }^{1}$ nous a confié la conduite d'une enquête sur ce sujet, dont le cahier des charges spécifiait : "confrontée à la mobilisation intense des médecins libéraux généralistes puis spécialistes et à la détermination avec laquelle ils se sont exprimés à plusieurs reprises depuis 2002, la CNAMts a souhaité initier une recherche qui lui fournisse une meilleure compréhension de cette situation de crise, aboutissement des ruptures traversées depuis un certain nombre d'années par la profession de médecin généraliste et des difficultés des institutions représentant et organisant ces médecins pour les accompagner à travers ces ruptures.»

Cette recherche nous a permis d'étudier ce secteur de la médecine et de constater que les médecins libéraux cherchent à s'exonérer des régulations qu'on veut leur imposer. Dans ce travail, nous ne nous sommes pas tant attachés aux régulations économiques, même si elles sont un enjeu de poids, préférant les systèmes d'action concrets aux modèles abstraits, donc fictifs des économistes ; mobilisant les acteurs institutionnels et macro-sociaux (l'Ordre, les caisses d'assurance maladie, les syndicats de médecins, les ministères de la santé et du budget, etc.) étudiés par la science politique, mais aussi les acteurs individuels et leurs comportements, à quelque niveau qu'ils soient situés : local, régional ou national.

Nous nous proposons de comprendre comment un groupe d'acteurs qui revendiquent fortement leur autonomie personnelle arrive toutefois à créer un collectif actif, en vue de conduire leurs intérêts professionnels. Il nous faudra pour cela examiner les dispositifs de régulation concernant les médecins libéraux afin de montrer les stratégies qu'ils mettent en œuvre pour les contrecarrer, sachant qu'aucun mot d'ordre n’a jamais été lancé dans ce sens par un acteur institutionnel du système.

\footnotetext{
1 Convention «Représenter, Organiser, Réguler l'exercice de la profession de médecin libéral » de la CNAMts du 19 août 2003.
} 
Aussi mobiliserons-nous le corpus de la science politique pour comprendre le contexte historique de la médecine libérale française dans ses relations houleuses avec les institutions et nous tirerons quelques conclusions sur le caractère propre du libéralisme dont se prévalent ces médecins. Puis nous éclairerons plus finement, grâce à l'enquête de terrain citée supra, les mécanismes de contrôle des régulations institutionnelles du système d'action, tel qu'il peut être appréhendé en ce début de siècle.

\section{Une méthode d'observation qualitative}

Le cadre théorique de notre enquête est celui de la sociologie des organisations qui s'attache à décrire les logiques d'acteurs et leurs interactions afin de définir le système d'action concret qu'ils forment et les régulations qui les régissent effectivement (Crozier \& Friedberg, 1977).

Le recueil des données est qualitatif, par entretien individuel semi directif long (deux heures). Il s'agit de se mettre à l'écoute des acteurs dans une posture compréhensive. L'anonymat est observé. L'échantillon n'est donc pas « statistiquement représentatif », mais raisonné : nous fixons un certain nombre de critères descriptifs. Ici, nous voulions recueillir les propos de médecins libéraux plutôt jeunes (moins de 45 ans) ${ }^{2}$ donc moins fatigués que leurs aînés par l'astreinte de la permanence des soins (PDS) qui consiste à répondre aux demandes des malades la nuit et le week-end pour assurer la continuité des soins. Nous voulions aussi interviewer un tiers de spécialistes et deux tiers de généralistes qui peuvent ne pas avoir les mêmes syndicats.

Pour analyser un système, il faut tenir compte de la dimension relationnelle et donc examiner dans le champ d'étude des "grappes relationnelles" afin de pouvoir étudier des interactions. Nous avons donc opéré quatre forages « institutionnels et territoriaux », du national au local. Nous avons choisi quatre régions dans chacune desquelles nous avons retenu le ressort d'une seule Caisse Primaire d'Assurance-Maladie (CPAM) qui correspond le plus souvent à un département, pour y interroger des médecins libéraux (14 généralistes et 7 spécialistes).

Les quatre CPAM ont été choisies en fonction de la densité en médecins libéraux du département (ce critère permet de dénombrer les médecins installés et de rapporter le chiffre à la moyenne nationale. Il permet d'évaluer la pénurie ou la pléthore au regard de cette moyenne) : région " $\mathrm{A}$ " faible densité (fortement rurale) $71^{3}$; " $\mathrm{B}$ ” faible densité (rurale, une grande ville et quelques moyennes) 73; “C" densité moyenne (urbaine) 99; "D" forte densité (choix d'un secteur urbain très dense) 150. Au total, nous avons rencontré 59 généralistes et 24 spécialistes, soit 83 praticiens.

L'approche qualitative permet des observations fines. Mais pour généraliser, il faut pouvoir comparer, c'est pourquoi l'analyse développée ici est fondée sur quatre monographies d'objets comparables que l'on cherche à contraster afin de relever les considérants semblables et dissemblables. La comparaison doit permettre de construire un idéal type, éventuellement des variantes, voire une typologie. Or, nous avons constaté une très grande

\footnotetext{
${ }^{2}$ Ce critère répondait à une demande du commanditaire.

${ }^{3}$ Nombre de médecins libéraux pour 100000 habitants; source : Base Eco-santé de I'IRDES.
} 
convergence des propos des acteurs dans les quatre régions étudiées, ce qui ne nous a pas permis de contraster nos monographies, sauf de façon marginale avec la quatrième, " $D$ " concernant un secteur à haute densité de médecins : 150 généralistes pour 100000 habitants $^{4}$, ce qui est nettement supérieur à la moyenne nationale (89) et ne met pas les médecins dans un contexte de pénurie comme dans les autres régions investiguées.

L'un des premiers problèmes exprimés par les médecins était celui de la surcharge de travail et à travers lui, l'aspiration à des modes de vie calés sur ceux du salariat. Les seules différences avec le secteur à haute densité tenaient à ce que les médecins trouvaient assez facilement un point d'équilibre entre vie professionnelle et vie privée, en raison de la régulation opérée par le marché qui se charge pour eux de fournir un nombre adéquat de patients (équilibre offre/demande). Par ailleurs, les relations avec la CPAM n'étaient pas tendues.

Notre échantillon global comporte 163 entretiens au niveau local, régional et national, dont 83 médecins en exercice, 60 institutionnels infranationaux et 20 nationaux (annexe 1).

Comme annoncé, nous allons maintenant nous attacher à décrire le système d'action des libéraux et ses régulations. Les acteurs : l'Ordre; les syndicats; la Sécurité sociale. Les enjeux : les stratégies d'action ainsi que leurs effets.

\section{Le système d'action des médecins libéraux face à la mise en place d'une gouvernance par la Sécurité sociale}

Le modèle économique traditionnel de la médecine libérale française sera largement bouleversé par l'apparition progressive d'un nouvel acteur : l'assureur. En 1945-1946 les premières caisses d'assurance de la sécurité sociale sont mises en place et constituent un enjeu idéologique et symbolique très fort : après les déchirements dus à la guerre, il faut réconcilier les Français et instaurer un pacte de solidarité entre eux (pacte toujours actif de nos jours). C'était aussi l'occasion de chercher à sortir des antagonismes de classe et de mettre les partenaires sociaux en position de coopérer pour assurer le financement et l'animation de cette nouvelle institution. La Sécurité sociale va permettre d'établir une relation contractuelle et responsable entre les représentants du Travail et du Capital.

Au fil du temps, le caractère local des Caisses (Galant, 1955) va se perdre et les Caisses nationales des différentes branches vont centraliser leur fonctionnement. La gouvernance va naturellement reposer sur la conception et la pratique antérieure de la négociation syndicale des salariés. Toutefois pour ce qui concerne la branche maladie, qui seule concerne notre sujet, un autre acteur doit nécessairement être introduit dans les négociations : le corps médical. Or celui-ci a toujours été hostile aux assurances sociales (Tabuteau, 2009).

On aurait pu imaginer que la Caisse aurait en face d'elle des organisations représentatives de la profession faisant office de courroie de transmission, en capacité d'encadrer leurs mandants, comme c'était le cas avec les syndicats de salariés du vingtième siècle telle la CGT (Andolfatto, Labbé D., 1997) ou pour les agriculteurs la FNSEA (Luneau, 2004). Or cela sera loin d'être le cas pour les médecins libéraux, en raison notamment de l'évolution socio-historique agitée de leur profession en relation avec l'État, dont voici les faits saillants.

\footnotetext{
${ }^{4}$ Chiffre donné par la CPAM locale.
} 


\section{Une petite histoire institutionnelle de la profession médicale française}

La Révolution française en abolissant les corporations bouleverse le système médical antérieur et va jusqu'à supprimer les structures universitaires considérées comme des corporations. Puis, deux ordres médicaux seront créés : celui des docteurs en médecine, ayant obtenu le grade de docteur et celui des officiers de santé ayant obtenu un brevet. Moins instruits que les docteurs, ils ne seront autorisés à pratiquer les grandes opérations chirurgicales que sous la surveillance et l'inspection d'un docteur.

Toutefois leurs effectifs, dans un premier temps, vont se développer beaucoup plus vite que ceux des docteurs (Tabuteau, 2009), même s'ils sont mal considérés, car ils constituent une alternative positive à la prolifération des guérisseurs qui sévissent notamment dans les campagnes médicalement abandonnées (Steffen, 1987).

$\mathrm{Au}$ seuil du $\mathrm{XIX}^{\mathrm{e}}$ siècle, la Convention vote une loi pour mettre en place un système d'assistance publique et envisage de recruter des médecins cantonaux salariés. Ces derniers auraient dû soigner les indigents chez eux, gratuitement; mais aussi faire fonction d'épidémiologistes et de conseillers en matière d'hygiène publique. Par manque de moyens, ce projet ne vit jamais le jour (Steffen, 1987).

Outre la préférence de la profession pour les soins à domicile et non à l'hôpital, cette époque pose trois principes essentiels de l'éthique médicale : le libre choix du médecin par le malade, le secret médical absolu et la liberté thérapeutique permettant au médecin de prescrire en fonction de sa seule conscience, sans en rendre compte à aucune instance (Steffen, $1987: 20-22$ ).

C'est toutefois "le début d'un malentendu historique sur lequel se sont établies, en France, les relations entre le corps médical, du moins dans sa composante libérale, et les pouvoirs publics. Car cette opposition latente, qui s'exprime parfois de manière exacerbée lors des négociations d'une nouvelle convention médicale, explique largement et sous-tend l'organisation actuelle du système de soin » (Tabuteau, 2009).

Les syndicats médicaux émergent à partir de 1881 parallèlement au développement des mutuelles, favorisées par les lois de 1852 et 1898 . Ils sont d'abord locaux puis ils se réunissent au sein de l'Union des syndicats médicaux français (USMF) (Steffen, 1987 : 25) mais leur reconnaissance légale est refusée par la Cour de cassation en 1885 (Hassenteufel, 2008).

Il faut attendre 1892, la loi Chevandier, pour que les officiers de santé soient supprimés et que le monopole de l'exercice de la médecine par les docteurs soit institué. Cette loi légalise également les syndicats professionnels de médecins. L'État intervient ici en leur faveur (Tabuteau, 2009) faisant une totale volte-face historique.

Au sortir de la Grande Guerre, dans les années 1920, la profession connaît de nombreux conflits internes et avec l'État. En décembre 1920, elle va faire pour la première fois une grève administrative en relation avec la question du tarif.

Une charte libérale rédigée par le docteur Paul Cibrie ${ }^{5}$ sera imposée au Congrès des syndicats médicaux de France le 30 novembre 1927 (Steudler, 1977), « charte qui

${ }^{5}$ Paul Cibrie fut longtemps président de la CSMF avant d'en devenir son secrétaire général en 1929, puis son président après la seconde guerre mondiale (Hassenteufel, 2008). 
continue de faire figure de texte de référence pour le syndicalisme médical français » (Hassenteufel, 2008).

Le caractère libéral s'exprime tout particulièrement à travers les quatre principes de la charte médicale : libre choix du médecin par le malade; liberté de prescription du médecin; entente directe entre malade et médecin en matière d'honoraires; paiement direct des honoraires par le malade au médecin. Ces principes ont été repris par le Code de déontologie, publié par le Journal Officiel en 1947, augmenté du principe de respect absolu du secret professionnel (Steudler, 1977). La création de l'Ordre des médecins est demandée dès 1920 par la CSMF, mais ne sera décidée, par décret, qu'en 1940 avec le régime de Vichy (Hassenteufel, 2008).

Les années 1950 seront marquées par une série de conflits et une crise qui amène le Ministre du Travail, en 1960, à imposer par décret, sans aucune négociation, un nouveau système : le principe du conventionnement collectif départemental qui permettra de ne pas trop bousculer les principes libéraux, de négocier le tarif et d'autres avantages comme la retraite, etc.

La première convention nationale fut signée en 1971 et, selon Steudler (1977) « $d u$ fait de l'implication plus grande du CNPF dans la gestion de la Sécurité sociale va faire évoluer le syndicalisme des médecins, de type corporatiste, vers un syndicalisme professionnel comprenant l'utilité d'une autodiscipline » (op .cit. p. 190-193). L'enjeu de ces négociations était la construction d'une régulation négociée (Hassenteufel, Pierru, 2003 : 102) du système de soins avec ses acteurs à tous les niveaux,6 alors qu'avec la centralisation de la CNAMts, le niveau national pesait très lourd sur la conduite des affaires.

Selon Hassenteufel (1997 : 179), "les limites de la régulation paritaire sont apparues nettement dans le contexte de la mise sur agenda de la maîtrise des dépenses de santé, qui devient, à partir des années 1980, la priorité de l'action publique dans le secteur ».

La conclusion de ce parcours historique institutionnel peut se résumer à deux caractéristiques prégnantes :

- d'une part, des relations très pugnaces entre l'État et les médecins libéraux qui, malgré leur capacité à constituer des organisations collectives de représentation, ne se départissent pas d'un individualisme viscéral, ou « coutumier» (Arliaud \& Robelet, 2000). Ainsi, en 1960, le Ministre du travail en jouera-t-il pour forcer la main à la CSMF en autorisant chaque libéral à signer individuellement la convention-type, ce qui fut suffisant pour que "le syndicat signe le texte de crainte d'un désaveu de fait par les praticiens. » (Arliaud \& Robelet, 2000).

- d'autre part, un refus tout aussi viscéral et coutumier à rendre des comptes à quiconque, comme le relèvent un certain nombre d'observateurs académiques de cette profession : « Le libre choix du médecin par le malade, le secret médical absolu et la liberté thérapeutique permettant au médecin de prescrire en fonction de sa seule conscience, sans en rendre compte à aucune instance » (Steffen, $1987: 22)$.

${ }^{6}$ "La régulation négociée ou paritaire est prise dans un sens proche de celui d'auto-administration, expression qui caractérise le mode de régulation du système d'assurance-maladie allemand » (Hassenteufel, 1997). 
Pour J. P. Dupuy \& S. Karsenty, cités par Steudler, 1977 : 185, le secret « signifie le refus de tout contrôle extérieur sur l'activité du médecin et non comme on pourrait le penser, sur l'état du malade».

«Dire que la profession médicale est une profession libérale, c'est, selon nous, non seulement mettre l'accent sur l'autonomie intellectuelle, mais sur le fait que chaque membre est en quelque sorte son propre maître, jouit d'un statut proche de celui de l'entrepreneur libre, les règles professionnelles garantissant alors cette indépendance morale et économique » (Steudler, 1977).

Enfin on peut noter que cette profession se complaît dans la plus grande ambiguïté :

«Une profession avec et contre l'État» pour (Steffen 1987). À la fois pour l'État, lorsqu'il établit les conditions de reconnaissance des docteurs en médecine et qu'il crée ainsi un monopole de soins; avec l'État encore, lorsqu'il régule les entrants dans la profession au moyen du numerus clausus, barrière d'entrée voulue par la profession et sa «peur récurrente de la pléthore » (Hassenteufel, 2008).

Mais elle est contre l'État lorsqu'il cherche à faire rendre des comptes et à réguler l'exercice des praticiens. Il y a certainement un lien à faire entre cette ambiguïté et la nature du concept de docteur libéral. Comme le précisent (Arliaud \& Robelet, 2000) : "Le libéralisme traditionnel de la profession n'est toutefois pas un libéralisme proprement économique. »

"La médecine libérale française renvoie à un ensemble de notions et d'attitudes assez floues. Elle est avant tout un "concept symbolique", une idéologie utilisée comme instrument de combat dans un affrontement social. Elle s'est forgée au fur et à mesure que l'Etat libéral français mettait en place, lentement, un système de protection sociale. L'assurancemaladie a permis un compromis politique entre l'État et les médecins » (Steffen, 1987 : 31).

Si, au fil de l'histoire, la profession reste attachée à la liberté sans entrave de sa pratique, son incapacité à supprimer le lien du patient à une assurance l'oblige à composer avec une contrainte qui, finalement, lui est économiquement favorable. C'est pourquoi la dynamique historique tend à mettre en place une régulation négociée entre l'assurance et la profession. C'est le fonctionnement contemporain de cette régulation que nous allons maintenant examiner au regard de l'enquête de terrain que nous avons menée.

\section{La production de la régulation négociée}

Comme nous l'avions annoncé en introduction, le management de la CNAMts consiste à animer la vie contractuelle, notamment avec les médecins libéraux. Les médecins doivent donc se constituer en groupes habilités pour la négociation. Ce qui suppose la mise en place d'un certain nombre de dispositifs plus ou moins complexes.

\subsection{La construction de la représentativité syndicale}

Tout comme pour les syndicats de salariés, leur représentativité doit être établie. La direction de la Sécurité sociale au Ministère de la Santé est chargée de la procédure. Avant l'échéance de la convention nationale en cours, une enquête de représentativité est menée à la demande expresse des organisations syndicales afin d'établir les syndicats qui seront habilités à négocier la prochaine convention pour les cinq ans à venir. 
Les critères sont les suivants :

- Les effectifs sont examinés ainsi que le nombre de cotisants. Mais les syndicats n'ont pas tous la même définition des effectifs : pour certains, il faut avoir cotisé pendant six mois, pour d'autres c'est un an, etc. Toutefois, une norme, non contestée, issue d'une jurisprudence du Conseil d'État, fixe le seuil minimum de la représentativité de la profession à $5 \%$ des effectifs des médecins.

- L'indépendance morale et économique est le second critère investigué. On examinera par exemple si cette organisation est proche d'un laboratoire pharmaceutique. Puis l'expérience du syndicat sera prise en compte : ses publications, son implication antérieure dans des négociations.

- La Direction Régionale des Affaires Sanitaires et Sociales de l'Île de France (tous les sièges sociaux de ces organisations sont en Île de France) constituera un dossier et se rendra au siège social. Le dossier sera soumis à la Direction de la Sécurité sociale. Il appartiendra au Ministre de déclarer tel syndicat représentatif ou non. " $C$ 'est une démarche très empirique » qui fait toutefois l'objet d'une publication au Journal Officiel. En définitive, c'est le Ministre de la santé qui accrédite les interlocuteurs des Caisses maladie. Il peut y avoir un caractère discrétionnaire dans ce choix. L'Union Collégiale des Chirurgiens et Spécialistes Français (UCCSF), constituée en 1992 a obtenu la représentativité dès 1997 afin qu'elle puisse signer une convention spécifique pour les médecins spécialistes (Robelet, 2003 : 216); en 1984 le Syndicat de la Médecine Générale, ancêtre de MG France, n’obtient pas la reconnaissance de sa représentativité, malgré l'avis favorable de l'enquête (Hassenteufel, 1997 : 164, 233).

Les syndicats que nous avons pu rencontrer ont confirmé avoir systématiquement subi cette procédure avant toute invitation à négocier la convention; respectivement :

- La Confédération des Syndicats Médicaux Français (CSMF), qui réunit deux collèges, l'Union Nationale des Omnipraticiens Français pour les généralistes et l'autre pour les spécialistes. C'est le syndicat historique des libéraux (1928), signataire des premières conventions avec les Caisses en 1971 et 1993... (Hassenteufel, 1997 : 93)

- Le Syndicat des Médecins Libéraux (SML) créé en 1981 en réaction à l'élection de M. Mitterrand, pour défendre le secteur 2, réunit aussi généralistes et spécialistes ;

- Médecins Généralistes de France (MG France), syndicat de généralistes (1985), se reconnaît "un engagement politique social de gauche" ; reconnu représentatif en 1989 (Tabuteau, $2006: 179$ ).

- Le Syndicat National des Jeunes Médecins Généralistes (SNJMG), créé en 1991, vise les jeunes généralistes mais n'a toujours pas été reconnu comme représentatif.

Tous ces syndicats reconnaissent leur difficulté à évaluer leurs effectifs et à faire remonter au niveau national l'argent collecté au niveau départemental. Cette difficulté comptable est une constante du syndicalisme, y compris salarial. Rosanvallon (1998 : 33, 261-262) a montré que cette démarche, hétérogène, crée des artefacts statistiques qui produisent des adhérents quantitatifs supérieurs aux adhérents physiques.

Les enjeux de cette reconnaissance, outre le fait de représenter la profession, sont d'accroître leur visibilité et leur audience auprès de leurs confrères, mais aussi d'être systématiquement invités aux réunions et donc informés des affaires en cours. C'est encore de pouvoir accéder à des sources de financement à travers la formation de leurs militants et la formation médicale continue. Cette formation conventionnelle, financée par les 
Caisses, est un moyen indirect de soutenir l'existence matérielle des syndicats. Elle a coûté 14,17 millions d'euros en 2001 selon le rapport d'activité de l'Organisme Gestionnaire pour la formation professionnelle conventionnelle (OGC, 2001) ce qui est peu au regard des 2,1 milliards dépensés en 2001 par Les Entreprises du Médicament pour la diffusion de leur propre information (LEEM, 2001).

Les syndicats déclarés « représentatifs » semblent donc très dépendants des Caisses et en premier lieu de la CNAMts. La régulation négociée est-elle donc réellement une régulation opérante? C'est ce que nous allons examiner dans les points suivants en rapportant les comportements et les attitudes des acteurs : médecins libéraux de base, puis interlocuteurs conventionnels nationaux et enfin infranationaux.

\subsection{La réputation des syndicats représentatifs des libéraux}

Deux types d'acteurs sont en mesure de porter des jugements sur les syndicats de médecins : d'une part leurs mandants et, de l'autre, leurs partenaires.

\section{Les médecins libéraux critiques de leurs syndicats}

Les médecins jugent sévèrement leurs syndicats qui ne sont pas crédibles. Ils ne font pas confiance à leurs confrères. Ils leur reprochent d'une part d'être financés par les Caisses d'assurance-maladie, d'autre part de les compromettre en acceptant de rentrer dans des grandes problématiques économiques et technocratiques et enfin de ne pas s'occuper des problèmes concrets : "ce sont des notables qui ont oublié les problèmes du terrain ». Lors de l'enquête, la plupart des médecins interrogés ne se souvenaient pas du nom du syndicat pour lequel ils avaient voté ou cotisé. Il y a de leur part une volonté de délégitimation de leurs représentants dont la dynamique peut être résumée en trois moments :

- tout d'abord la régulation négociée voulue par la CNAMts passe par la signature de conventions. Les syndicats de médecins manquent cruellement de moyens, y compris de militants, comme nous avons pu le constater dans les départements et les régions. Les Caisses doivent donc aider leurs interlocuteurs;

- ensuite les médecins connaissent ces mécanismes et reprochent à leurs organisations d'être « vendues » : peut-on être financé par la puissance qui vous fait obligation de négocier? Veut-on participer à une technocratie centralisatrice qui ne s'adapte pas aux problèmes locaux? Les médecins ont aussi en tête un autre modèle de représentativité, celui de la démocratie de proximité qu'animent les Coordinations qui ont fleuri à partir de 2001. Elles jouent sur le bénévolat et l'entraide sociale, se centrent sur les problèmes de proximité et diffusent une information immédiate à tous les médecins, mais sont exclues des négociations ;

- enfin, les syndicalistes "représentatifs" se sentent en porte-à-faux. Ils assument alors la coupure avec leur base et cherchent même à la renforcer afin de trouver une légitimité supérieure : problèmes nationaux et de long terme; langage des économistes (panier de soins, etc.) que leurs confrères refusent de comprendre. Les militants les jugent mal informés et peu actifs. Du coup, ils s'auto-mandatent : "C'est mon AG qui me mandate, pas l'ensemble des médecins. » Ils tournent le dos à leurs confrères, la coupure est consommée. Elle n'est voulue par personne en particulier, mais elle est produite par tout le monde en général. Le paradoxe 
de cela c'est le mécanisme discréditant d'accréditation constitué par la décision ministérielle qui rend possible les financements par l'Assurance-maladie et contribue à miner la représentativité syndicale.

\section{Évaluation des syndicats par leurs partenaires de négociation}

La représentativité des syndicats de médecins libéraux est elle-même non assumée par la quasi-majorité des responsables syndicaux. Dans les instances dirigeantes de la CNAMts, un syndicaliste chevronné, déclare : "À mon arrivée ici, il m'est arrivé d'entendre dire : "ma signature ne m'engage pas"; j'étais interloqué. Aujourd'hui, je pense sincèrement que leur signature ne les engage pas ». Nous avons nous-mêmes rencontré des responsables syndicaux de médecins qui nous ont tenu le même langage. Il faut savoir que les médecins ne sont pas favorables à des signatures de convention et sont très réactifs lorsque leurs dirigeants le font. Ils cessent de cotiser ou de voter pour les syndicats signataires. Cela est arrivé en 1996 par exemple lorsque MG France a signé en faveur de la mise en place du médecin référent qu'il préconisait lui-même (Hassenteufel, Pierru, 2003 : 101) : cela lui a valu de perdre plus de la moitié de ses électeurs du collège généraliste aux élections de 2000 (Tabuteau, 2006 : 195).

MG sera mis à nouveau en porte-à-faux en 2001, lors des négociations pour le passage de la Consultation à vingt euros (C à $20 €)$. MG France sera cette fois-là d'autant plus déconsidéré par les médecins libéraux que Jacques Chirac, en campagne électorale, apporte son soutien aux autres syndicats et accédera à leur demande quelques mois après son élection (Tabuteau, $2006: 232$ ).

Lors de notre enquête qui se déroulait pourtant quatre ans plus tard, nous avons constaté combien les militants de MG France étaient stigmatisés par leurs collègues. Le président de MG nous a déclarés : "notre essence même est de négocier le contrat. À chaque fois que l'on signait un accord que l'on pensait bon, on ne comprenait pas pourquoi il était rejeté par les professionnels. La C à $20 €$ nous a fait comprendre qu'à chaque fois qu'on signait un accord, on rendait visible "l'asservissement de la profession à la Sécu". Ils nient la solvabilisation du marché ».

Les libéraux ne veulent pas de compromis avec l'Assureur et sont prêts à affaiblir les syndicats qui ne suivent pas cette stratégie.

\subsection{La construction du collectif}

Au-delà de l'offre syndicale de ces professionnels, très souvent mobiles et abstentionnistes, deux grandes familles se constituent qui orientent différemment le poids et le respect de la régulation négociée.

\section{Deux stratégies syndicales en présence}

Lorsque le Président de MG France parle de l'essence de l'action de son syndicat, il est totalement en phase avec la présidence de la CNAMts, c'est-à-dire en accord avec l'action contractuelle négociée. Il exprime aussi son sentiment que ses adhérents ont des convictions convergentes sur la santé, le rôle du médecin, qu'ils acceptent l'autorité des dirigeants de leur syndicat et qu'ils mettent en œuvre les clauses contractuelles. Cette idéologie est le ciment du groupe qu'il représente. Or, cette vision est loin d'être partagée par les praticiens, d'autant que les conventions signées “ouvrent droit" sans s'imposer à eux puisqu'ils restent toujours libres de ne pas être conventionnés. 
Les autres syndicats, tout en participant au jeu conventionnel, cherchent à faire passer leurs idées tout en ne signant pas, ou très discrètement. Ils cherchent aussi à rencontrer le cabinet du Ministre et à contourner la CNAMts pour obtenir des avantages sans contreparties.

Lors du passage de la consultation à vingt euros (C à $20 €)$, il y a eu une intrusion du pouvoir politique dans le dispositif de régulation négociée. Toutefois, l'État est tout à fait légitime à intervenir, compte tenu de ses prérogatives en matière de santé publique, affirmées lors de la réforme de la loi sur le numerus clausus en 1979.

Il y a donc deux stratégies en œuvre : celle que l'on peut appeler du Conventionnalisme, qui relève du paradigme de la régulation négociée, supposant des acteurs solidaires entre eux et respectueux d'une autorité morale, engagée dans un collectif, conforme à la solidarité mécanique (Durkheim, 1922) et une autre stratégie que l'on peut appeler de Lobbying. Cette dernière consiste, par exemple, à faire casser par le Conseil d'État le dispositif de reversement d'honoraires dépassant les prévisions annuelles votées par le parlement, prévu par le Plan Juppé : Objectif National des Dépenses d'Assurance-maladie (ONDAM). Puis par faire censurer par le Conseil Constitutionnel le nouveau dispositif législatif voté à l'initiative du Gouvernement Jospin, dans le cadre du projet de loi de financement de la sécurité sociale pour 1999 en mobilisant 60 députés et 60 sénateurs de l'opposition pour qu'ils puissent saisir le Conseil constitutionnel en vue de le confirmer ${ }^{7}$.

Or, il y a une différence de taille entre ces deux stratégies qui porte pour la première sur un engagement effectif des représentés à se soumettre au contrat passé et pour la seconde, à une prévalence de l'intérêt individuel.

Nous rejoignons là les analyses de Rosanvallon (1998) sur le déclin contemporain du syndicalisme. Les médecins viennent d'entrer encore d'avantage dans l'individualisme professionnel du fait du désajustement produit par la pénurie de praticiens, qui a rempli leur salle d'attente, les mettant en suractivité. Ils se sont épuisés au travail et ont commencé à sélectionner leurs patients, à tenir plus strictement leurs horaires, diminuer les jours d'ouverture et prendre des vacances. Ils sont passés d'une vision de leur profession, vécue comme un apostolat, à une autre dans laquelle ils se projettent dans une société moins coercitive.

C'est ce que notre enquête nous a permis de constater sur le terrain. Cette évolution professionnelle est confirmée par Soulier, Grenier, Lewkowicz et al. (2005). Les médecins, dans leur grande majorité, se positionnent explicitement dans le cadre d'une société individualiste et non plus solidaire.

\section{Une régulation négociée, mais peu légitimée}

Que penser de cette régulation négociée? Nous relevons une neutralisation forte de la représentativité syndicale, assortie de boycotts des syndicats signataires qui décrédibilisent leurs représentants et dénient le système même de la régulation négociée.

Nous relevons l'intrusion des hommes politiques dans les négociations et donc dans la régulation des partenaires qui dénie, elle aussi, cette existence et, renforce la propension des syndicats à faire du lobbying pour chercher à contourner le cadre conventionnel. Il est alors peu surprenant que la grande majorité des médecins que nous avons interviewés aient décrit leur horizon institutionnel comme largement borné par une CPAM jugée « technocratique » et par un Ordre, CDOM (Conseil Départemental de l'Ordre des Médecins), qu'ils

\footnotetext{
${ }^{7}$ Le Quotidien du médecin, 21 décembre 1998.
} 
brocardent, mais fréquentent au moins pour s'y inscrire et qui se rappelle à eux, via les cotisations annuelles obligatoires.

À l'autre extrême, ils connaissent le nom de leur Ministre qu'ils estiment être un recours puissant (surtout lorsqu'il est médecin) sur lequel ils reposent des espoirs, souvent déçus, d'obtenir un soutien. Mais entre le niveau départemental et national, il y a un vide gigantesque. Ils disent même ne pas connaître L'Union Régionale des Médecins Libéraux (URMEL) alors qu'ils sont censés y élire des représentants de leurs syndicats. Plus largement, les libéraux ne connaissent pas ou peu les divers organismes du niveau régional, voire départemental.

La stratégie collective des médecins renforce donc la faiblesse des syndicats. Bien entendu, personne n'a ourdi cette stratégie ; là encore, il s'agit d'un effet de système, construit collectivement par agrégation de réactions et d'évaluations de situations singulières et dispersées ; "solidarité naturelle de la classe professionnelle » dirait Freidson ${ }^{8}$; mais dispersion qui converge pour produire ce résultat global. Les free-riders sont majoritaires et mettent à mal l'idée d'une régulation négociée efficace.

Ces comportements sont très importants car ils sont rendus possibles du fait de leur inscription dans le contexte d'action de proximité des professionnels (on est loin de Paris, des pressions exercées pour modifier des textes juridiques ou réglementaires). On est là au niveau micro, celui des comportements que l'on espère tant, au niveau macro, transformer par des règles nouvelles. C'est à ce niveau que nous allons nous intéresser en revenant à la vie conventionnelle locale, puis à l'Ordre et enfin aux médecins conseils des CPAM.

\subsection{La vie conventionnelle locale}

La vie conventionnelle locale désigne tout d'abord, dans le contexte institutionnel de l'Assurance-maladie, la fixation négociée au niveau national avec les syndicats représentatifs des médecins, de la tarification des actes médicaux, etc. Son suivi doit être fait par les CPAM dans le cadre de la Commissions Conventionnelle Paritaire locale (CCPL) et de la Commission Médicale Paritaire Locale (CMPL). La première (CCPL) comporte des responsables administratifs de la CPAM et des représentants syndicaux des libéraux. L'Ordre y est présent à titre consultatif. Cette commission permet de commenter localement et de décliner les engagements conventionnels nationaux. Par exemple, la mise en œuvre de l'informatisation des cabinets et les subventions afférentes. La seconde (CMPL) ne comporte que des médecins : praticiens des Caisses et représentants des libéraux. Ici, on ne débat que de problèmes médicaux ou liés aux comportements des médecins. Par exemple, l'annonce d'une campagne d'entretiens confraternels sur le diabète. Cette animation est peu suivie par les médecins libéraux. Mais surtout, il ressort de notre enquête qu'en réalité cette vie est ténue, voire inexistante. Les quatre monographies que nous avons réalisées permettent de montrer les difficultés empiriques rencontrées.

Région A : Le département connaît une Coordination «exemplaire ». La vie locale est très conflictuelle et les médecins rejettent globalement la CPAM. Cela évacue toute vie conventionnelle au niveau départemental et même régional. Les leaders de la Coordination

\footnotetext{
${ }^{8}$ Freidson op. cit. p. 355.
} 
"s'invitent chez le directeur de la CPAM lorsqu'ils le souhaitent »; ils ont par ailleurs leurs entrées à l'Ordre, à l'Union Régionale des Médecins Libéraux (URMEL) et sont affiliés à divers syndicats nationaux. MG est complètement déconsidéré et le syndicat départemental s'est effondré au passage de la consultation à vingt euros $(\mathrm{C}$ à $20 €)$.

Au niveau régional, il n'y a pas de coopération avec son interlocuteur institutionnel, l'Union Régionale des Caisses d'Assurances Maladie URCAM réunissant la CNAMts (régimes des salariés), la CANAM (artisans-commerçants) et la MSA (Agricoles). La Coordination (non habilitée à négocier) a fait une proposition à la CPAM pour organiser la mission de permanence des soins, mais l'URCAM a refusé car elle ne peut payer aux conditions demandées, suivant en cela des consignes nationales.

Région B : La vie conventionnelle départementale est inexistante depuis plusieurs années, mais en voie de reprise lors de notre venue avec le retour de MG France et celui de SML; pour les spécialistes, il n’y a plus aucun partenaire libéral dans les Commissions locales et régionales.

Région C : Il a existé une Coordination de généralistes, mais elle est en déclin, faute d'avoir pu rebondir sur la crise de la PDS (département urbain avec un Centre 15 et SOS médecin). Comme elle n'a pas de moyens, l'Association voit ses bénévoles (i.e. la Coordination) s'essouffler et les leaders se sont égayés dans des syndicats nationaux différents. Seul MG siège dans les CCPL, mais cela est mal perçu par les confrères des autres syndicats qui le stigmatisent : «Ce sont des collabos ». CSMF est présente en force à l'URMEL mais ne signe pas de conventions.

Région D : Dans le département, le syndicat dominant est la CSMF. Il dispose aussi de la quasi-totalité des postes à l'Ordre. Les partenaires se connaissent depuis longtemps. Les syndicats et l'Ordre n'arrivent pas à mobiliser leurs confrères. Aussi y a-t-il un très petit nombre de médecins : quatre en tout qui se retrouvent dans toutes les instances pour participer aux niveaux local et régional. Il existe aussi une coordination. Les problèmes de PDS sont extrêmement prégnants. Les généralistes font la grève des gardes; l'Ordre ne veut plus fournir les tableaux d'astreinte et les médecins sont réquisitionnés par la Direction Départementale de l'Action Sanitaire et Sociale (DDASS).

Pour conclure cette revue de la vie conventionnelle infra nationale, on constate qu'elle ne brille pas par sa dynamique et que les acteurs n'arrivent pas ou mal à coopérer, ce qui se voit notamment au regard de la gestion de la PDS qui est prise en main, soit par un syndicat ou une coordination ou par l'Ordre comme il lui incombe normalement, soit encore par le Préfet lorsqu'il y a carence de son organisation. Mais c'est toujours par un seul groupe qui écarte les autres. Comme le dit avec regret le président d'un conseil d'administration d'une CPAM «il ne reste plus rien aujourd'hui des négociations avec les professions de santé ».

La vie conventionnelle locale et régionale n'est pas très active, les syndicats ne voulant pas, voire ne pouvant pas y participer pleinement faute de participants. La régulation négociée au plus proche des médecins ne fonctionne donc pas non plus, au mieux empêchée, notamment par le centralisme parisien, le manque de représentants syndicaux et le refus quasi général du paradigme conventionnaliste.

Cette régulation est tellement difficile à produire qu'il a fallu mettre en place un dispositif juridique pour ne pas arrêter les soins : en l'absence de convention, un Règlement Conventionnel Minimal (RCM) est fixé par arrêté ministériel, qui s’inspire de la précédente 
convention et les règles sont fixées unilatéralement. Par exemple, en 2003, la convention des généralistes a été remplacée par un RCM, les spécialistes ont bénéficié d'un RCM pendant sept ans de suite ${ }^{9}$.

Autant dire que la convention négociée est caduque puisqu'elle est unilatéralement établie. Mais pire encore pour la légitimité du paradigme conventionnaliste, il faut rappeler que les dernières élections des cotisants aux conseils d'administration de la Sécurité Sociale ont eu lieu en 1983, puis ont été supprimées par la suite.

Toutefois, la régulation conventionnelle négociée n'épuise pas les accords sur les dispositifs de régulation locaux, régionaux et nationaux. En effet, un certain nombre de régulations concernent tous les libéraux dans leur vie quotidienne, qu'elles soient organisées par l'État seul, l'Ordre seul, ou les assurances sociales elles-mêmes. Il nous reste à en décrire le fonctionnement et à en évaluer l'efficacité.

\section{Les régulations individuelles des professionnels}

Comme nous l'avons précisé dès l'introduction, l'Ordre des médecins est institutionnellement la première instance de régulation des professionnels français ; mais ils peuvent aussi avoir affaire avec l'assureur par le truchement des médecins conseils de la Sécurité sociale et par celui de son tribunal administratif (TASS).

\subsection{La régulation ordinale}

La mission de l'Ordre des médecins comporte cinq grands chapitres : un rôle moral, un rôle administratif (l'Ordre dispose d'un pouvoir réglementaire), un rôle juridictionnel, un rôle consultatif (il est en particulier appelé à donner son avis sur les projets de règlements, décrets ou de lois qui lui sont soumis par les pouvoirs publics) et enfin, un rôle d'entraide (www.conseil-national.medecin.fr, 8 décembre 2003). Mais la structure de fonctionnement de l'Ordre n'est pas toujours bien connue des praticiens. Son instance régionale nouvellement implantée, le Conseil Régional de l'Ordre des Médecins (CROM), n'est pas très lisible. Les médecins qui y avaient été convoqués, suite à une plainte (les Chambres disciplinaires de première instance y étant situées) le connaissaient, mais ne s'en vantaient pas. Lors des entretiens, lorsque le sujet de l'Ordre était abordé, la plupart des médecins commençaient d'y répondre en rapportant une petite anecdote qu'ils avaient eux-mêmes vécue ou dont ils avaient été témoins dans leurs rapports avec l'Ordre, afin d'illustrer le décalage qu'il entretient avec les praticiens. Par exemple, déconseiller aux jeunes généralistes d'utiliser un téléphone portable et recommander de faire accepter à l'épouse de faire le secrétariat.

Quel que soit le niveau institutionnel, national, régional ou départemental, l'image du Conseil de l'Ordre n'est pas bonne et relève plutôt du jugement comportemental que de la référence à ses missions.

Notre corpus d'entretiens des médecins nous livre les thématiques qualificatives suivantes :

- « empoussiéré, vieux, arriéré, âgé, décalé, désuet, réactionnaire »;

- « copains, en vase clos, opaque, mystérieux »;

${ }^{9}$ Devenu Règlement arbitral depuis 2004. 
- « rigides, tatillons, assis sur leurs positions »;

- «pas dynamiques, toujours un train de retard»;

- «pusillanimes, ce qui caractérise l'Ordre, c'est qu'il ne prend jamais position, il se planque derrière des prétextes vagues ».

Au niveau national, l'Ordre donne des avis. Il fait des audiences qui sont désormais publiques, ce qui a modifié son action, car le public et la presse peuvent en faire état. Au niveau régional, ils sont jugés très conservateurs et rétifs aux médecins marginaux qui nuisent à l'image de la profession. Par exemple "un médecin homosexuel sera cassé » (un généraliste). Au niveau départemental, «l'Ordre aide ses confrères à débrouiller un problème professionnel » (un généraliste).

Les reproches plus politiques faits à l'Ordre par les praticiens portent sur trois points principaux :

$\mathbf{1}^{\circ}$ Une mauvaise gestion des dossiers : les médecins notent son incapacité à faire aboutir les choses, par exemple la mise en place du fichier des remplaçants, ou celui du statut du collaborateur libéral.

$2^{\circ}$ Une incapacité à se projeter dans l'avenir. Par exemple, il n'a pas vu venir la crise de la PDS, ni anticipé le problème, alors qu'il savait que les trois quarts des médecins s'en exonéraient facilement avec des structures de type SOS Médecin. Si bien qu'il s'est fait imposer par l'État la modification de l'article 77 du Code de déontologie qui ne fait plus obligation aux médecins d'assurer l'astreinte de nuit et de week-end qui relève dorénavant du volontariat.

Ce n'est pas la première fois qu'il y a eu une intrusion dans les affaires de l'Ordre, comme nous l'avons vu précédemment. Mais cet épisode touchant à la déontologie touche au cœur de sa légitimité. Comme nous l'avons déjà dit : avant la convention nationale de 1971, la fixation des honoraires était du ressort de la déontologie «La sécurité sociale elle-même n'est qu'une intrusion dans les missions de l'Ordre des médecins » (Ministère, Direction de la Sécurité sociale).

$3^{\circ}$ L'Ordre n'arrive pas à faire front aux partenaires institutionnels : Comme nous venons de le constater, l'Ordre est souvent marginalisé par l'État, mais il se fait aussi déborder par des syndicalistes.

Par exemple, en 2002, le Président du CSMF envoie un tract demandant d'annuler l'obligation des gardes et provoque ainsi une crise au sein de l'Ordre dont le président démissionne. Un membre des plus hautes instances de la CNAMts commente : "Avec la fin de l'obligation des gardes, je crois que quelque chose de symbolique a sauté et qu'il y a une déliquescence de l'Ordre ». Lors de notre enquête, on nous a signalé, dans plusieurs départements étudiés, qu'il y avait une confusion et un malaise entre l'Ordre et certains "syndicats majoritaires qui ont les mêmes locaux et les mêmes secrétaires ». Il semble bien que si le monde des libéraux voulait entamer l'autorité de l'Ordre, il ne s'y prendrait pas autrement.

L'Ordre des médecins ne semble plus être « une institution qui porte les choses » mais cela est bien normal si cette institution n'est plus portée par la profession qu'elle représente et doit contribuer à réguler. En effet comme l'explique (Lagroye, 2008) à propos de la hiérarchie catholique vue de France " C'est en définitive l'appréciation portée sur les dirigeants par leurs pairs et par les autres membres du groupe qui assure le maintien de la légitimité. Comme l'a fortement dit La Boétie "ce sont les sujets qui font les princes" ». 
Sera-ce le cas aussi pour l'un des derniers acteurs de la régulation non encore évoqué jusqu'ici : le médecin-conseils? Il nous reste à le confirmer.

\subsection{La régulation assurancielle : Médecins-Conseils, TASS et Ordre}

Les médecins-conseils sont le bras armé du contrôle opéré pour réguler l'exercice professionnel des médecins libéraux. Une ordonnance de 1967 (CNAMts, 2003) a rattaché le Service du contrôle médical directement à la Caisse nationale : les médecins-conseils sont hiérarchiquement indépendants de leurs structures logistiques, CPAM, CRAM, CNAM. Ce service comporte 2500 médecins-conseils et 7500 agents administratifs. Les médecinsconseils s'appuient pour leurs contrôles sur des argumentaires et des recommandations établis par des médecins dans le cadre d'agences : Agence Française de Sécurité Sanitaire des Produits de Santé (AFSSAPS), Agence Nationale d'Accréditation en Santé (ANAES) ou par la Haute Autorité de Santé (HAS) ${ }^{10}$.

Les médecins-conseils sont essentiellement installés dans les services médicaux des CPAM. Ils sont loin d'être chaleureusement accueillis par les praticiens qui les nomment souvent « méd-cons ». Ils estiment que ce se sont des bureaucrates qui ne pratiquent pas et qui viennent contrôler des confrères alors qu'ils ne se risquent même plus à diagnostiquer. Les recommandations des Agences aident les médecins-conseils à s'exonérer de ce genre de commentaires.

L'objectif du Service Médical est d'œuvrer pour une meilleure qualité des soins, au regard des recommandations. Il est donc dans une logique de protection et d'amélioration pour les assurés sociaux : "On joue au nom des assurés ». A la différence de l'Ordre, ils ne sont pas là pour réguler une profession libérale, mais un système de soins, et pour veiller aux bonnes pratiques. Ils interviennent aussi pour signaler des erreurs de facturation (cotation) et les pratiques médicales peu orthodoxes, voire répréhensibles. "Si un médecin traitant fait n'importe quoi (d'ailleurs il est connu pour ça dans le coin), bien sûr, il faut intervenir! Le but c'est aussi de conforter les professionnels vertueux. Si on ne dit rien sur les abuseurs, cela désespère les vertueux » (Direction du Service médical).

Dans chaque CPAM il existe un dispositif coercitif : la Commission Médicale Paritaire Locale (CMPL). Lorsqu'un médecin vient à être suspecté, une enquête est diligentée. Le Service du contrôle médical, par exemple pour un abus de cotation, va transmettre ses conclusions au médecin suspecté par la Caisse. Cette dernière a la possibilité de récupérer les indus et discute avec le Service médical pour une éventuelle saisine du Tribunal Administratif de la Sécurité Sociale (TASS). Si cette pratique est récurrente, alors elle peut être considérée comme une faute déontologique et ressortir du CROM. C'est le Service médical qui fournira les éléments de preuve et le respect de la procédure. Deux cents saisines environ sont faites annuellement en France.

Toutefois le Service du contrôle médical est loin d'être satisfait par les sanctions infligées : "prenons l'exemple d'un chirurgien orthopédique qui bousille ses malades. On lance une procédure et puis au bout de 5 ans, le Conseil de l'Ordre lui inflige un mois de suspension d'exercice avec sursis. C'est vraiment se moquer de nous! Ou encore, il y a une élection présidentielle et le médecin est gracié. Le gars continue parce qu'il sait qu'il ne risque rien! » (direction nationale du Service médical).

${ }^{10}$ L'ANAES a été intégrée par la HAS en 2004. 
La régulation par les médecins-conseils comporte différentes instances et différents degrés d'action (de faible à fort) : entretien confraternel, convocation à la CMPL, au TASS et juridiction ordinale. De fait, toutes les affaires sérieuses doivent nécessairement passer par l'Ordre et si les procédures sont lourdes, les sanctions sont légères et rares. Seuls «les cas indéfendables et patents» sont sanctionnés. Ils ne semblent concerner que $5 \%$ de déviants lourds (chiffre plusieurs fois donné oralement et jamais démenti). Or ces sanctions ne sont pas lourdes et donc pas en mesure de faire évoluer les comportements moyens. Le système de soins semble bien, comme on nous l'a dit maintes fois, non régulé. Il existe pourtant une mesure simple à mettre en œuvre par les CPAM qui est le déconventionnement du médecin s'il ne prend pas en compte les résultats du contrôle qu'il a subi.

Or, le déconventionnement est, nous a-t-on dit, "impensable. C'est une arme atomique que l'on ne peut utiliser sous peine d'embraser la profession ». Il est certain qu'il manque donc une panoplie d'actions graduées, plus légères et rapides, au service des médecinsconseils, afin de faire respecter les recommandations des Agences. La sanctuarisation est donc une stratégie de neutralisation parmi d'autres, nous y reviendrons.

Le lieu d'exercice de cette panoplie semble exister avec les CMPL, qui ne sont pas des instances contentieuses, mais qui font se rencontrer des notables ${ }^{11}$ " syndicalistes qui ont oublié les problèmes du terrain » et "Conseillers ordinaux qui, au niveau du département, débrouillent un problème professionnel ». On s'y arrange entre soi, au mieux, éventuellement on dirigera le cas sur une instance disciplinaire (CROM) ou juridique (TASS). Reste aux CCPL à orienter les praticiens épinglés.

L'Ordre qui maîtrise la dimension juridictionnelle est incontournable pour la régulation du système. "Le législateur a voulu, en effet, que les médecins puissent être jugés et éventuellement sanctionnés par leurs pairs qui connaissent bien les problèmes soulevés par les malades et par l'exercice de la profession ». Le rôle juridictionnel s'exerce par l'intermédiaire des conseils régionaux en première instance et en appel par la section disciplinaire du Conseil National, présidé par un Conseiller d'État, membre de l'Ordre. Ce pouvoir résulte des dispositions du code de la santé publique; il est contrôlé, en ce qui concerne la légalité de ses décisions, par le conseil d'État, instance de cassation ${ }^{12}$. L'Ordre est conscient des limites qu'il impose au cadre juridictionnel, mais semble s'en satisfaire car son objectif est de protéger les médecins, même défaillants.

«Toute plainte est instruite. Elle va au Conseil régional qui donne la sanction. Le médecin n'est pas content, il fait appel. La sanction est confirmée, elle va au Conseil d'État. Il est arrivé que l'on soit cassé. C'est lourd et long, mais c'est rassurant pour les médecins »(CNOM).

Paradoxalement, l'image ringarde et décalée de l'Ordre, transmise par les médecins libéraux, conforte sa puissance dilatoire. L'Ordre est perçu comme une grosse machine opaque et pusillanime qui cherche à étouffer le temps pour rendre possible l'oubli : une stratégie de l'oubliette.

${ }^{11}$ La force du notable, c'est d'accéder directement aux lieux de décision locaux, évitant ainsi les procédures lourdes et permettant les arrangements discrets. Worms, 1966; Crozier \& Friedberg, 1977.

12 Les missions de l'Ordre, chapitre Rôle juridictionnel. Ordre National des Médecins www.conseilnational.medecin.fr, décembre 2003. 


\section{Conclusion : une stratégie collective de neutralisation des régulations}

Lorsque nous reprenons les conclusions partielles de nos analyses, nous pouvons noter que tous les dispositifs que nous avons examinés sont neutralisés à des degrés divers :

- totalement pour le déconventionnement des médecins et la liberté d'installation, elle aussi sanctuarisée (Schweyer, 2006), ainsi que pour l'ONDAM victime d'un lobbying bien conduit auprès des parlementaires;

- très fortement pour la régulation négociée et la vie conventionnelle, quel que soit son niveau de localisation. Le Numerus clausus qui a fait l'objet d'une thèse remarquée (Déplaude, 2007) dont l'auteur montre très méticuleusement que son pilotage a toujours été dominé par le lobby médical. Enfin le conseil de l'Ordre lui-même en raison de sa stratégie de l'oubliette et les syndicats qui sont pour la plupart entravés dans leur signature, au demeurant de mauvais aloi. Seule la régulation locale par les notables est acceptée et non entravée. Tous les acteurs des régulations, quels qu'ils soient, sont discrédités : syndicats, Ordre, Caisses maladies. Nous avons affaire à un déni généralisé de l'autorité.

Le système français de la médecine libérale n'est donc pas bien régulé, sauf à la marge et localement. Il suit toutefois le principe appliqué par les professions.

Nous faisons l'hypothèse que l'enjeu de cette stratégie des médecins français, tout comme celle des Américains, est celui de l'autonomie professionnelle totale et complète, qui peut déborder le strict cadre de l'exercice de la médecine, comme le montre le refus, en France, de renoncer à la liberté d'installation. Nous savons que les médecins libéraux ne veulent pas avoir à rendre de comptes, même si, dans chacune de ces nations, ils acceptent la régulation légère et discrète qui lui est propre, mais Freidson juge que cette régulation par les pairs n'est pas heureuse car elle ne permet pas à la profession d'être régulée.

"Dans le contexte de liberté d'action que confère l'autonomie professionnelle, le seul dispositif de contrôle qui soit caractéristique et qui corresponde aux normes de l'activité clinique ou de consultation est le boycottage personnel; mais il est, par son profil, plus proche à régler le travail à l'échelle des individus qu'à celle de la profession comme telle »(Freidson, 84 : 198).

En France, la régulation de la profession tente d'exister, mais nous avons montré que cette régulation est très particulière puisqu'elle est dérégulante. Les médecins sont globalement consensuels pour pratiquer le déni d'autorité. Aussi n'est-il pas étonnant de voir un ancien président de la CSMF, à l'annonce du retrait du mécanisme de reversement d'honoraires prévu par la loi sur l'ONDAM, déclarer : "Ce n'est pas parce que le Conseil constitutionnel s'est prononcé que le gouvernement a changé de logique. Notre marche est longue, nous poursuivons la "guérilla" »".

Le cas français montre que les institutions en capacité de gérer et de réguler la profession sont en réalité peu tentées de le faire, connaissant le discrédit que la profession porte au niveau institutionnel, qu'elle récuse le plus souvent en le qualifiant de «technocratie ». Ceci permet d'ailleurs de comprendre pourquoi la seule régulation acceptée est celle des pairs et des notables. En effet, ces modes de régulation américains et français sont tous deux informels, donc, par construction, non institutionnalisables.

\footnotetext{
${ }^{13}$ Le Quotidien du médecin, 21 décembre 1998.
} 
La stratégie des médecins consiste donc à désinstitutionnaliser l'encadrement de leur exercice professionnel; ce qui reste cohérent avec leur individualisme généralisé et leur exigence d'autonomie totale et sans reddition, qui revient à vouloir bénéficier du statut juridique d'irresponsabilité. Mais, au-delà de ce statut, il est un autre enjeu majeur pour les médecins : la pression des économistes de la santé dont ils rejettent les travaux mais dont ils ne peuvent ignorer ni la présence, ni l'insistance des modélisations précises, "toutes choses égales par ailleurs » alors qu'elles imprègnent leur environnement professionnel.

Nous en prenons pour preuve la parution d'un petit opuscule «Où va le système de Santé français? » (Claude Le Pen, André Grimaldi, 2010) qui permet la confrontation entre Claude Le Pen, président du collège des économistes de la santé et André Grimaldi, professeur de médecine de renom. L'économiste milite pour le développement d'une médecine gérée rationnellement alors que le médecin la rejette au titre de sa « conception marchande » (2010, p.102). Pour lui, toute comptabilité analytique porte en elle la base d'une reddition de compte, mais surtout oublie la dimension humaine de la médecine, comme le montre l'exemple développé à propos des soins palliatifs : " $70 \%$ des Français meurent à l'hôpital... Quelle est la réponse d'une société marchande transformant l'hôpital en "entreprise" et le médecin hospitalier en "agent économique" au problème posé par la prise en charge de la fin de vie? " (idem p. 89); nos deux débateurs ne sont pas sur le même registre, même s'il est bien question de soins médicaux. Leur confrontation débouche sur une controverse et installe la polémique. Mais les médecins préfèrent éviter les débats et cherchent à faire taire leurs porte-parole (Ordre et syndicats) grâce à leur stratégie de déni systématique afin de ne pas avoir à y répondre. Personne n'envisage que les impératifs de l'autre sont aussi légitimes et nécessaires que les siens propres, en vue de chercher un équilibre acceptable.

Ces deux enjeux, l'irresponsabilité juridique et le refus des pratiques gestionnaires, permettent, in fine, de comprendre pourquoi, lors de notre enquête, nous avons eu la surprise de constater la grande convergence des propos de nos interlocuteurs.

\section{Remerciements}

Je remercie tous ceux, anonymes ou non, qui m'ont aidé et encouragé pour ce travail. Je reste néanmoins le seul responsable des analyses.

\section{Bibliographie}

ANDOLFATTO D. et LABBÉ D., (1997). La CGT. Organisation et ambiance depuis 1945, La Découverte, Paris.

ARLIAUD M. \& ROBELET M., (2000). Réforme du système de santé et devenir du corps médical, Sociologie du travail 42 (1). 91-112.

CNAMts, (2003). Direction du Service Médical Commentaires sur les dispositions législatives et réglementaires relatives aux missions du service médical du régime général de l'assurance-maladie CNAMts, 15 p.

CNOM, (2003). www.conseil-national.medecin.fr

CROZIER M. \& FRIEDBERG E., (1977). L'acteur et le système, Le Seuil, Paris. 
DÉPLAUDE M.-O., (2007). L'emprise des quotas - les médecins, l'État et la régulation démographique du corps médical (1960 à 2000). Thèse pour l'obtention du doctorat de sciences politiques soutenue le 14 décembre 2007, Université de Paris 1.

DURKHEIM E., (1922). La division du travail social, Félix Alcan, Paris.

FREIDSON E., (1984). La Profession médicale, Payot, Paris.

FRIEDBERG E., (1993). Le pouvoir et la règle - Dynamique de l'action organisée. Le Seuil, Paris.

GALANT H., (1955). Histoire politique de la Sécurité sociale française 1945-1952, Armand Colin, Paris.

HASSENTEUFEL P., (1997). Le plan Juppé : fin ou renouveau d'une régulation paritaire de l'assurancemaladie ?, Revue de l'IRES 24, 175-189.

HASSENTEUFEL P., (2008). Syndicalisme et médecine libérale : le poids de l'histoire, Seve 18, 21-28.

HASSENTEUFEL P. et PIERRU F., (2003). De la crise de représentation à la crise de régulation de l'assurancemaladie In KERVASDOUÉ, 77-120.

HASSENTEUFEL P., (1997). Les Médecins face à l'État - Une comparaison européenne, Les Presses de Sciences-Po, Paris.

KERVASDOUÉ de Jean (dir), (2003). La crise des professions de santé, Dunod, Paris.

LAGROYE J., (2008). Projet de lettre sur le gouvernement de l'Église catholique en France, Confrontations.

LE PEN C. et GRIMALDI A., (2010). Où va le système de santé français? Ed. Prométhée, Bordeaux.

LEEM. Les entreprises du médicament. La recherche avance, la vie progresse. document de présentation institutionnelle émanant du site http://www.leem.org

LUNEAU G., (2004). La forteresse agricole - Une histoire de la FNSEA, Fayard, Paris.

OGC,(2001). Rapport d'activité de l'Organisme Gestionnaire pour la formation professionnelle Conventionnelle, 217 rue La Fontaine, 94120 Fontenay sous Bois.

PIERRU F., (2007). Hippocrate malade de ses réformes, Éditions du Croquant, Bellecombe-en-Bauges.

ROBELET M., (2003). La grande désillusion des médecins libéraux : entre la tentation de la division et l'espoir d'une unité retrouvée In KERVASDOUÉ, 199-232.

ROSANVALLON P., (1998). La question syndicale, Hachette, Paris.

SCHWEYER F.-X., (2006). Ni artisan, ni salarié - Le choix de l'installation en médecine générale libérale Actes du colloque de Rennes Approches sociologiques de la médecine générale : état des recherches récentes et perspectives, organisé par l'AFS et l'ENSP à Rennes les 8 et 9 juin 2006.

SOULIER E., GRENIER C., LEWKOWICZ M. et al. (2005). La crise du médecin généraliste : une approche cognitive de la profession. Revue Médicale de l'Assurance-maladie 36 (1).

STEFFEN M., (1987). Les médecins et l'État en France, Politiques et management public 5 (3), 19-39.

STEUDLER F., (1977). Médecine libérale et conventionnement, Sociologie du travail 19 (2), 176-198.

TABUTEAU D., (2009). L'avenir de la médecine libérale et le spectre de Monsieur Bovary, Droit Social 4.

TABUTEAU D., (2010). Les défis des professions de santé - Pouvoirs publics et professions de santé. Les tribunes de la santé $n^{\circ} 26$, Presses de Sciences-Po, Paris.

TABUTEAU D., (2006). Les Contes de Ségur - Les coulisses de la politique de santé (1988 - 2006). Ed. Ophrys, Paris. VALAT B., (2001). Histoire de la Sécurité sociale (1945-1967). L'État, l'institution et la santé, Economica, Paris. WORMS J.-P., (1966). Le préfet et ses notables, Sociologie du travail 3. 


\section{Annexe 1 - Échantillon}

\section{Médecins}

\begin{tabular}{|c|c|c|c|}
\hline Régions & Généralistes & Spécialistes & Total \\
\hline A & 16 & 7 & 23 \\
\hline B & 14 & 6 & 20 \\
\hline C & 15 & 6 & 21 \\
\hline D & 14 & 5 & 19 \\
\hline Total & 59 & 24 & $\mathbf{8 3}$ \\
\hline
\end{tabular}

Institutionnels infranationaux : régions et départements

\begin{tabular}{|c|c|c|c|c|c|}
\hline $\begin{array}{l}\text { Régions } \\
\text { Organismes }\end{array}$ & A & B & $\mathrm{C}$ & $\mathrm{D}$ & Total \\
\hline $\begin{array}{l}\text { Ministère des Aff. } \\
\text { Sociales } \\
\quad \text { DDASS }\end{array}$ & 1 & 1 & & 2 & 4 \\
\hline $\begin{array}{r}\text { CnamTS } \\
\text { CPAM } \\
\text { URCAM } \\
\text { CRAM }\end{array}$ & $\begin{array}{l}4 \\
1\end{array}$ & $\begin{array}{l}6 \\
1 \\
1\end{array}$ & $\begin{array}{l}6 \\
1\end{array}$ & $\begin{array}{l}5 \\
1\end{array}$ & $\begin{array}{c}21 \\
4 \\
1\end{array}$ \\
\hline $\begin{array}{l}\text { Ordre des } \\
\text { Médecins } \\
\\
\text { CDOM } \\
\text { CROM }\end{array}$ & 1 & $\begin{array}{l}3 \\
1\end{array}$ & 1 & 2 & $\begin{array}{l}7 \\
1\end{array}$ \\
\hline $\begin{array}{r}\text { Syndicats } \\
\text { URML } \\
\text { Dép } p^{\text {taux }} \\
\text { Coordinations }\end{array}$ & $\begin{array}{l}1 \\
2 \\
1\end{array}$ & $\begin{array}{l}2 \\
3 \\
1\end{array}$ & $\begin{array}{l}2 \\
2 \\
1\end{array}$ & $\begin{array}{l}2 \\
3 \\
1\end{array}$ & $\begin{array}{c}7 \\
10 \\
4\end{array}$ \\
\hline $\begin{array}{l}\text { Hôpitaux } \\
\qquad \text { Centre } 15\end{array}$ & 1 & & & & 1 \\
\hline Total & 12 & 19 & 13 & 16 & 60 \\
\hline
\end{tabular}




\section{Institutionnels nationaux}

\begin{tabular}{|lr|r|}
\hline Ministère de la Santé & Direction de la sécurité sociale & 2 \\
\hline Ministère du Budget & 6 Sous-Direction & 2 \\
\hline Cnam TS & Direction Générale & 2 \\
& Médecins Conseils Nationaux & 3 \\
\hline Ordre des Médecins & CNOM & 2 \\
& & 2 \\
\hline Syndicats & CSMF & 2 \\
& MG-France & 2 \\
& SML & 1 \\
\hline Revue Prescrire & SNJMG & 3 \\
\hline Total & & 1 \\
\hline
\end{tabular}

Soit 163 entretiens : Médecins libéraux = 83

Régions et départements $=60$

National

20 


\section{Annexe 2 : Sigles utilisés}

AFSSAPS Agence Française de Sécurité Sanitaire des Produits de Santé

ANAES Agence Nationale d'Accréditation En Santé, fusionnée à la HAS

CANAM CAisse Nationale d'Assurance-maladie des Professions Indépendantes (Artisans \& Commerçants)

CCPL Commission Conventionnelle Paritaire Locale

CDOM Conseil Départemental de l'Ordre des Médecins

CMPF Commission Médicale Paritaire Locale

CNAMts Caisse Nationale d'Assurance-maladie des travailleurs salariés

CNOM Conseil National de l'Ordre des Médecins

CNPC Conseil National du Patronat Français

CPAM Caisse Primaire d'Assurance-maladie

CRAM Caisse Régionale d'Assurance-maladie

CROM Conseil Régional de l'Ordre des Médecins

CSMF Confédération des Syndicats Médicaux Français

DDASS Direction Départementale de l'Action Sanitaire et Sociale

DRASSIF Direction Régionale des Affaires Sanitaires et Sociales d'Ile de France

FNSEA Fédération Nationale des Syndicats d'Exploitants Agricoles

HAS Haute Autorité de Santé

LEEM Les Entreprises du Médicament

MG France Médecins Généralistes de France

MSA Mutualité Sociale Agricole

OGC Organisme Gestionnaire pour la Formation Professionnelle Conventionnelle

ONDAM Objectif National des Dépenses d'Assurance-maladie

PDS Permanence Des Soins

RCM Règlement Conventionnel Minimal, devenu Règlement arbitral depuis 2004

SML Syndicat des Médecins Libéraux

SNJMG Syndicat National des Jeunes Médecins Généralistes

TASS Tribunal Administratif de la Sécurité Sociale

UCCSF Union Collégiale des Chirurgiens et Spécialistes Français

UNOF Union Nationale des Omnipraticiens Français, collège généraliste de la CSMF

URCAM Union Régionale des Caisses d'Assurance-maladie (régimes des salariés, artisans-commerçants \& agricole)

URMEL Union Régionale des Médecins Libéraux

USMF Union des Syndicats Médicaux Français 
